\title{
Simultaneous endocardial and epicardial high-resolution mapping of the human right atrial wall
}

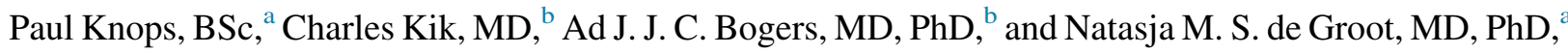 \\ Rotterdam, The Netherlands
}

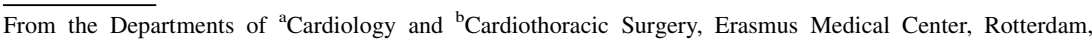
The Netherlands.

N.M.S.d.G. is supported by grants from the Erasmus Medical Center fellowship, Dutch Heart Foundation (2012T0046), LSH-Impulse Grant (40-43100-98-008), CoolSingel Foundation (No. 212), Bayer, and Boehringer Ingelheim.

Disclosures: Authors have nothing to disclose with regard to commercial support.

Received for publication March 29, 2016; revisions received May 4, 2016; accepted for publication May 9, 2016; available ahead of print June 17, 2016.

Address for reprints: Natasja M. S. de Groot, MD, PhD, Department of Cardiology, Erasmus Medical Center, PO

Box 616, 's Gravendijkwal 230, 3015 CE Rotterdam, The Netherlands (E-mail: n.m.s.degroot@erasmusmc.nl). J Thorac Cardiovasc Surg 2016;152:929-31

$0022-5223 / \$ 36.00$

Copyright (c) 2016 by The American Association for Thoracic Surgery

http://dx.doi.org/10.1016/j.jtcvs.2016.05.026
}

\section{Video clip is available online.}

In patients with long-standing persistent atrial fibrillation (AF), there is an increase in conduction disorders and a higher incidence of focal fibrillation waves compared with patients with induced AF. ${ }^{1}$ Prior mapping studies have demonstrated that the arrhythmogenic substrate underlying persistence of long-standing persistent AF can be identified by highresolution multisite epicardial mapping. ${ }^{1,2}$ From recent observations, we hypothesized that asynchronous activation between the endocardium and the epicardium promotes transmural conduction of fibrillation waves, which plays a key role in the pathophysiology of AF in humans. ${ }^{1,2}$ Simultaneous mapping of the endocardium and epicardium of the atrial wall is required to directly demonstrate endoepicardial asynchrony (Figure 1). In current electroanatomic mapping systems, the electrical information is recorded in a sequential manner, with low-resolution or noncontact mapping devices. Also, a stable rhythm is required, so they are not suitable for this task. Therefore, we developed a new high-resolution mapping technique to record electrical activation from both the endocardial and epicardial layers of the right atrial wall simultaneously.

\section{MATERIALS AND METHODS}

\section{High-Resolution Mapping}

Mapping studies, performed in our hospital, are part of the Quest for the Arrhythmogenic Substrate of Atrial Fibrillation (QUASAR) research program and have been approved by the Erasmus MC Medical Ethics Committee (MEC-2010-054). 3,4 Written consent was obtained from described. ${ }^{3,4}$ approach.

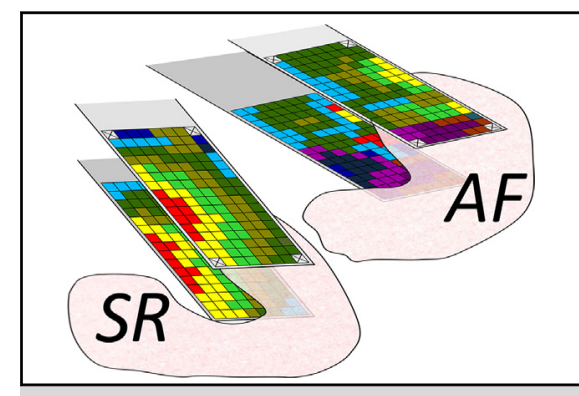

Schematic view of simultaneous endocardial and epicardial high-resolution mapping during sinus rhythm and AF.

\section{Central Message}

Coherence between endocardial and epicardial activation can be visualized by mapping. This example shows simultaneous activation in sinus rhythm and asynchronous activation during AF.

See Editorial Commentary page 932.

each participant. A clamp-shaped mapping device consisting of 2 equally thin, rounded, and bendable stainless-steel spatulas, containing 2 identical rectangular electrode arrays of $8 \times 16$ electrodes, was used for simultaneous mapping of the endocardial and epicardial side of the right atrial wall (Figure 2 and Video 1). The electrodes have a diameter of $0.65 \mathrm{~mm}$, and the inter-electrode distances are $2 \mathrm{~mm}$ in both directions. The surface of the area mapped is $14 \times 30 \mathrm{~mm}$, which equals $4.20 \mathrm{~cm}^{2}$. After heparinization and arterial cannulation, but before extracorporeal circulation, 1 leg of the mapping device was introduced through the right atrial auricle incision for venous cannulation and positioned onto the endocardium, opposite the second leg on the epicardial surface. The incision was temporarily closed using a purse-string suture. Good wall contact was achieved by clamping the 2 spatulas together. The identical dimensions of both endocardial and epicardial legs ensure accurate perpendicular alignment of the 2 electrode arrays. Analysis of the recorded endocardial and epicardial signals was performed as previously

In Figure 3, 2 pairs of endocardial and epicardial activation maps of a patient with a history of persistent AF are shown. Figure 3 demonstrates simultaneous endocardial and epicardial activation during sinus rhythm and dissociated activation of both layers during AF. The minimal differences in corresponding electrode local activation times during sinus rhythm confirm that an accurate alignment of the endocardial and epicardial mapping arrays is ensured by the use of the stainless-steel spatulas, and the remarkably bigger local activation time differences evolved during $\mathrm{AF}$ demonstrate that dissociation in conduction between the endocardial and epicardial layers can be visualized using this novel mapping 


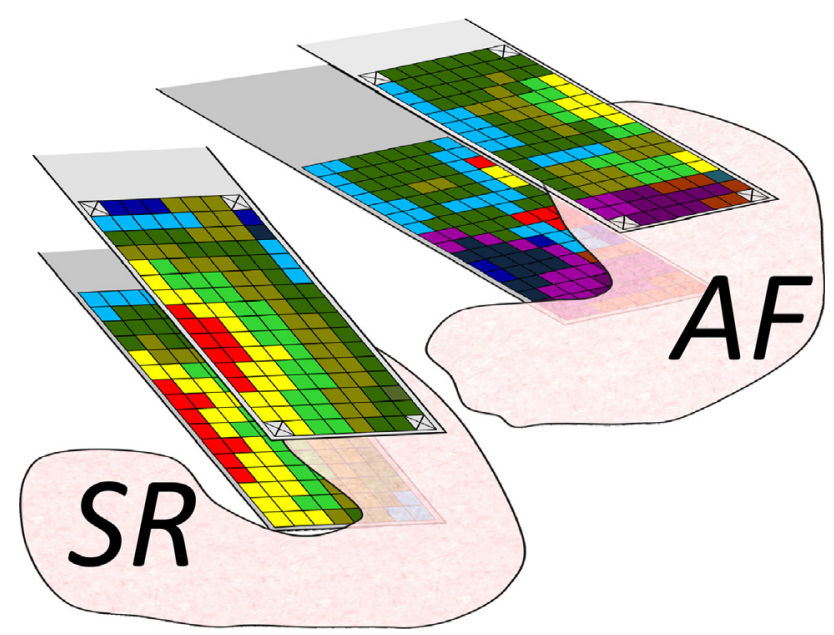

FIGURE 1. Schematic view of simultaneous endocardial and epicardial high-resolution mapping during sinus rhythm $(S R)$ and atrial fibrillation $(A R)$. Simultaneous endocardial and epicardial mapping is required to directly demonstrate synchronous activation between endocardium and epicardium.

\section{DISCUSSION}

Since 1991, epicardial mapping during open surgery has been a well-known technique for the evaluation of electrical properties of myocardial tissue. ${ }^{5}$ For research purposes on the electropathologic mechanism of AF, we developed a novel approach for simultaneous mapping on the endocardial and epicardial side of the right atrial wall. This modification was developed for further investigation on the electrical coherence between the endocardial and epicardial layers. The advantage of this mapping approach is that no new, proven, safe techniques were used. ${ }^{4}$ By applying this mapping approach, double layer information can be sampled simultaneously. However, mapping is limited to the area around the right atrial venous cannulation entrance site. Mapping of the interatrial septum or the left atrium during beating heart surgery is not possible yet without additional incisions and increased risk for complications. Currently, analysis of the recorded signals is not performed in real time, but when this becomes available, this tool might be valuable for guidance during Cox-Maze procedures.

\section{CONCLUSIONS}

Simultaneous epicardial and endocardial multisite high-resolution mapping offers advantages over epicardial mapping alone, allowing visualization of (a)synchronous activation of the atrial wall. This technique could improve diagnosis of the AF substrate and brings patient-tailored therapy of AF one step closer.
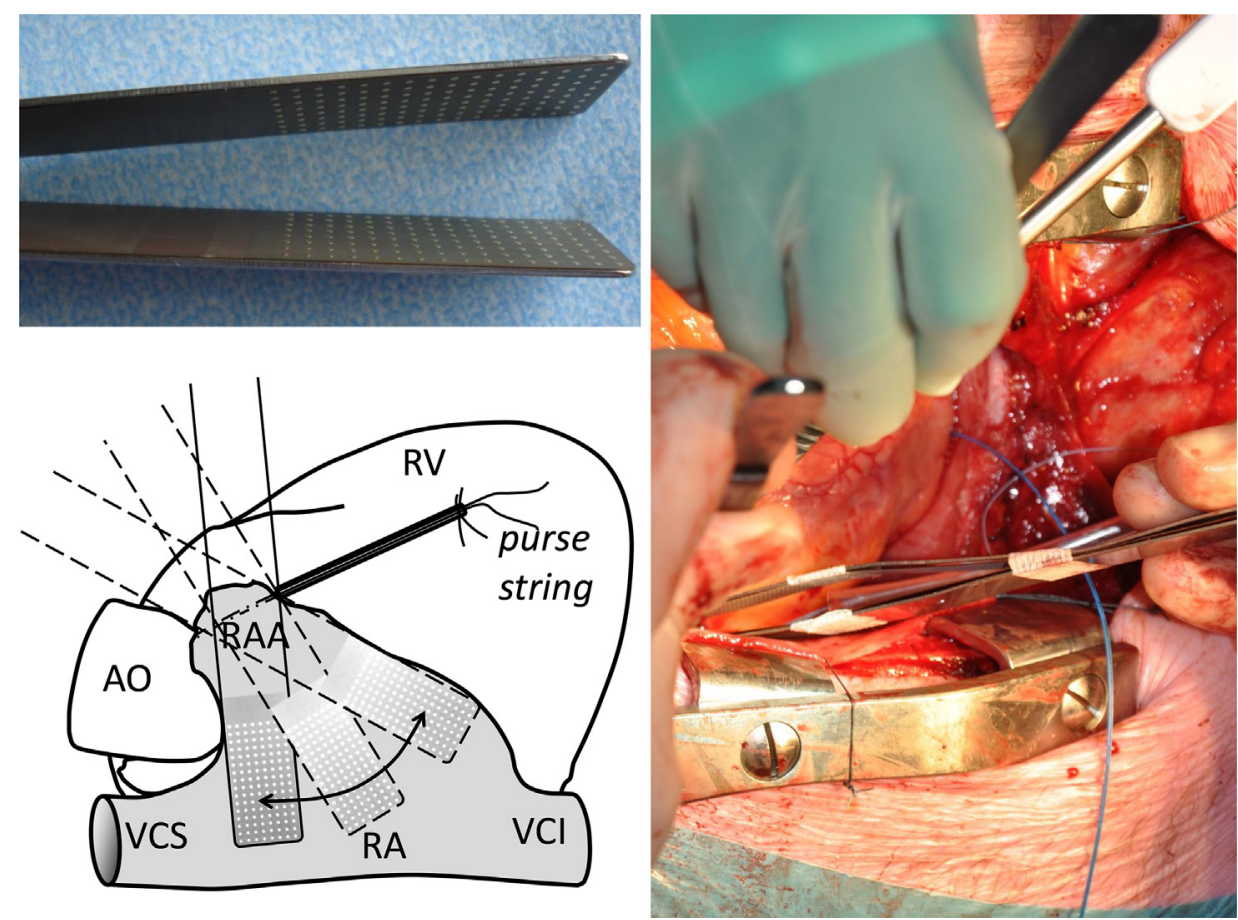

FIGURE 2. Mapping electrode array and endocardial/epicardial mapping clamp. Top left: Close up of 2 aligned 128 pole mapping arrays assembled on 2 thin, rounded, and bendable stainless-steel spatulas (perspective view); details are explained in the text. Bottom left: Diagram showing the mapping positions on the right atrial free wall that can be attained. Right: Intrathoracic view during insertion of the assembled mapping clamp. $R V$, Right ventricle; $A O$, aorta; $R A A$, right atrial appendage; $V C S$, vena cava superior; $R A$, right atrium; $V C I$, vena cava inferior. 


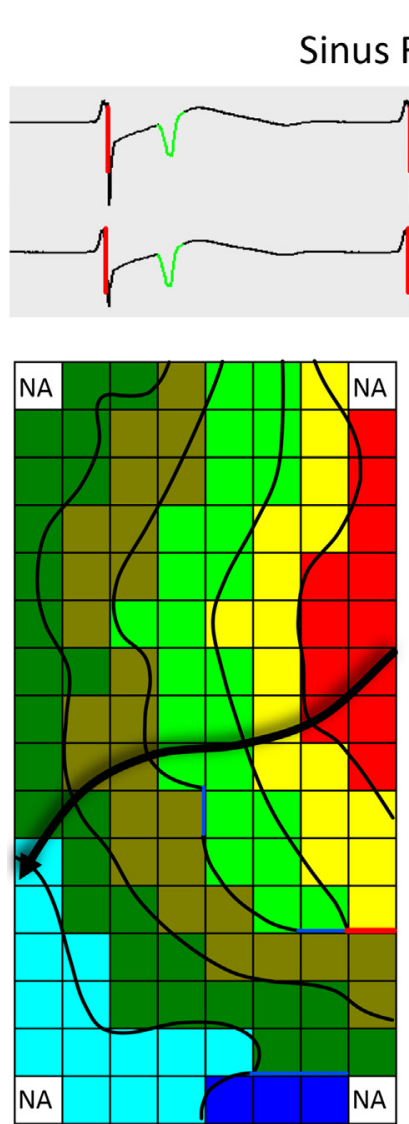

Epicardium

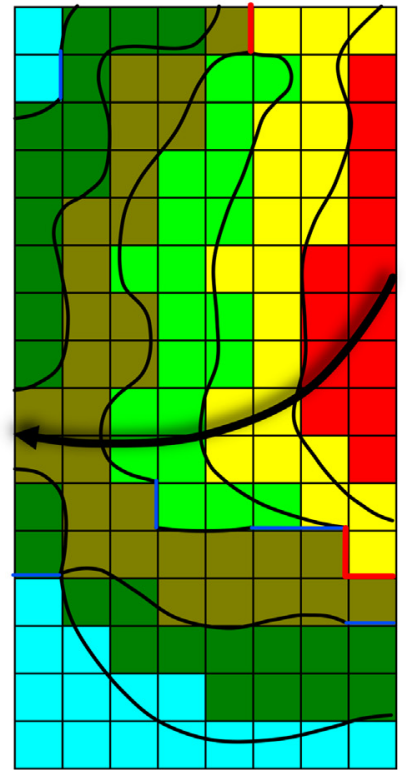

Endocardium

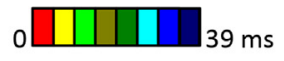

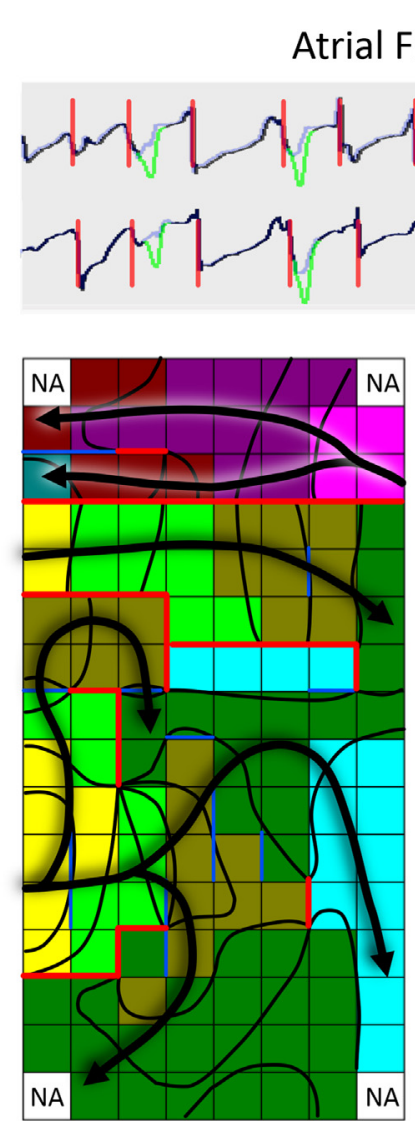

Epicardium

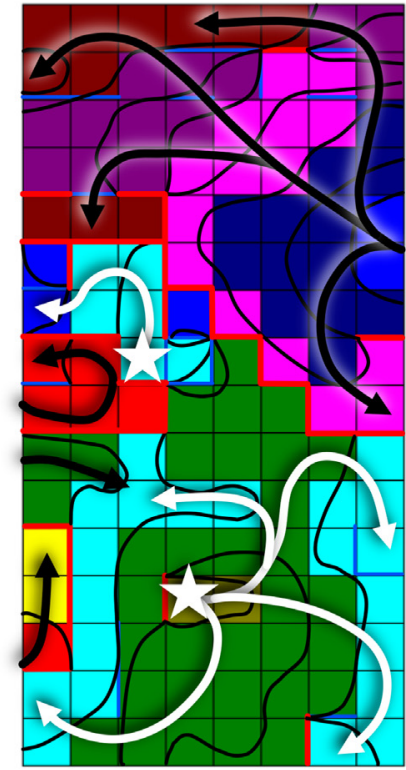

Endocardium

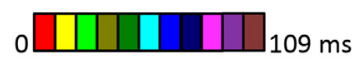

FIGURE 3. Examples of color-coded local activation time (LAT) maps during sinus rhythm (SR) and AF. These simultaneous endocardial and epicardial mapping data were achieved from a patient with a history of persistent AF during elective coronary artery bypass surgery. The electrode arrays are represented by 2 rectangular rastered areas in which each electrode is represented by a single raster box. A blue raster line visualizes a time delay of more than $7 \mathrm{~ms}$ between 2 contiguous electrodes, whereas a time delay of more than $11 \mathrm{~ms}$ is depicted as a thick red raster line. (The LAT numbers are removed from the maps for better visualization.) Thin black lines represent isochrones of LAT, drawn at 5 ms intervals. Thick black and white arrows indicate the main activation trajectories (black = peripheral wave: entering from outside of the mapping area, white = focal wave: arising from within the mapping area). Electrograms of an associated endocardial and epicardial electrode pair are shown in the rhythm strips above the maps (time frame 2 seconds; the epicardial electrogram is on top). The far-field ventricular activations are marked in green. The local atrial activations are automatically marked by the vertical red lines (For AF, the ventricular activation signal is subtracted from the signal before automatic marking of the atrial activation.) Left: Endocardial and epicardial LAT maps during SR. During SR, the endocardium and epicardium are almost simultaneously activated. The maps demonstrate accurate perpendicular alignment of both mapping electrode arrays. The endocardial and epicardial mapping area is activated within a time span of maximal 39 ms. Right: Endocardial and epicardial LAT maps derived during AF. Both endocardial and epicardial mapping areas show significantly more complex and dissociated activation patterns. The total time required to activate the complete area of both mapping electrode arrays is approximately 3 times longer than during SR (106 ms). The 2 sites marked with white stars indicate the earliest activation within the mapped area, which is suggestive for transmural wave propagation (white arrows). NA, Not available.

\section{References}

1. Allessie MA, de Groot NM, Houben RP, Schotten U, Boersma E, Smeets JL, et al. Electropathological substrate of long-standing persistent atrial fibrillation in patients with structural heart disease: longitudinal dissociation. Circ Arrhythm Electrophysiol. 2010;3:606-15.

2. de Groot NM, Houben RP, Smeets JL, Boersma E, Schotten U, Schalij MJ, et al. Electropathological substrate of longstanding persistent atrial fibrillation in patients with structural heart disease: epicardial breakthrough. Circulation. 2010;122:1674-82.
3. Yaksh A, Kik C, Knops P, Roos-Hesselink JW, Bogers AJJC, Zijlstra F, et al. Atrial fibrillation: to map or not to map? Neth Heart J. 2014;22:259-66.

4. Yaksh A, van der Does LJ, Kik C, Knops P, Oei FB, van de Woestijne PC, et al. A novel intra-operative, high-resolution atrial mapping approach. J Interv Card Electrophysiol. 2015;44:221-5.

5. Cox JL, Canavan TE, Schuessler RB, Cain ME, Lindsay BD, Stone C, et al. The surgical treatment of atrial fibrillation. II. Intraoperative electrophysiologic mapping and description of the electrophysiologic basis of atrial flutter and atrial fibrillation. J Thorac Cardiovasc Surg. 1991;101:406-26. 